05. 1;06.5; 13.3

\title{
Механические напряжения и гигантский пьезоотклик поверхности текстурированной керамики $\mathrm{K}_{2} \mathrm{Sr}_{4} \mathrm{Nb}_{10} \mathrm{O}_{30}$
}

\author{
() М.А. Бунин, О.А. Бунина, Ю.А. Куприна, В.П. Завьялов \\ Научно-исследовательский институт физики Южного федерального университета, Ростов-на-Дону, Россия \\ E-mail: bunin.m.a@gmail.com
}

Поступило в Редакцию 14 мая 2019 г.

В окончательной редакции 21 мая 2019 г.

Принято к публикации 21 мая 2019 г.

\begin{abstract}
Кристаллографические характеристики керамических образцов $\mathrm{K}_{2} \mathrm{Sr}_{4} \mathrm{Nb}_{10} \mathrm{O}_{30}$ сопоставлены с данными о пьезоотклике поверхности, полученными методом сканирующей зондовой микроскопии, на основании которых оценены относительные величины пьезомодулей $d_{33}$. В зависимости от ориентации поверхности керамического образца относительно оси текстуры они в 62 или 58 раз превышают значение для изотропного образца. Причиной гигантского пьезоотклика поверхности могут быть анизотропные деформации решетки, вызванные действием давления при горячем прессовании. Обсуждается роль доменных границ и полярных нанообластей.
\end{abstract}

Ключевые слова: тетрагональная вольфрамовая бронза, пьезоотклик поверхности, текстура, $\mathrm{K}_{2} \mathrm{Sr}_{4} \mathrm{Nb}_{10} \mathrm{O}_{30}$.

DOI: 10.21883/PJTF.2019.16.48157.17876

Одноосные сегнетоэлектрики со структурой тетрагональной вольфрамовой бронзы (ТВБ) являются перспективной основой для бессвинцовых активных материалов нового поколения [1,2]. Технология горячего прессования (ГП) позволяет эффективно создавать в них аксиальную текстуру с преимущественной ориентацией осей $c$ игольчатых кристаллитов перпендикулярно оси давления $[3,4]$. Ниобат калия-стронция $\mathrm{K}_{2} \mathrm{Sr}_{4} \mathrm{Nb}_{10} \mathrm{O}_{30}$ (KSN) со структурой ТВБ обладает высокими диэлектрическими, электромеханическими и электрооптическими характеристиками [5-8], которые обнаруживают зависимость от технологии получения [9]. Это может быть связано с различием микроструктуры получаемого вещества, а также с влиянием добавок, используемых при синтезе в расплаве солей, и испарением летучих компонентов в процессе синтеза. В технологии ГП эти факторы сведены к минимуму. В настоящей работе проведен сравнительный анализ структурных и микроструктурных характеристик керамики KSN, приготовленной с помощью метода ГП и обычной керамической технологии.

Полученный в результате ГП керамический блок характеризуется плотностью более $98 \%$ от теоретически возможного значения и степенью аксиальной текстуры 45\%. Измерительные образцы KSN_N $\|$ P и $\mathrm{KSN} \_\mathrm{N} \perp \mathrm{P}$ вырезались в двух различных ориентациях относительно оси текстуры (рис. 1). Контрольный изотропный образец KSN_iso получен с помощью обычной керамической технологии с использованием двухстадийного синтеза. Режимы синтеза и спекания керамики детально описаны в $[9,10]$. Рентгенодифракционные исследования выполнены на дифрактометре Rigaku UltimaIV, $\mathrm{Cu} K_{\alpha}$-излучение. Обнаруженные в горячепрессованной керамике остаточные деформации приводят к понижению температуры фазового перехода на $25^{\circ} \mathrm{C}$ по сравнению с таковой для $\mathrm{KSN}$ iso. Совместный анализ рентгенодифракционных данных двух измерительных образцов KSN_N $\|$ P и KSN_N $\perp$ P позволил определить параметры тетрагональной решетки для $a$ - и $c$-ориентированных кристаллитов (см. таблицу и рис. 2). Для образца $\mathrm{KSN} N \mathrm{~N} \| \mathrm{P}$ отражения $(h k 0)$ формируются $a$-ориентированными кристаллитами, а отражения $(00 l)-c$-ориентированными (рис. 2). Для образца $\mathrm{KSN} \_\mathrm{N} \perp \mathrm{P}$ отражения $(h k 0)$ формируются $c$-ориентированными кристаллитами, а отражения $(00 l)-a$-ориентированными. Исходя из полученных значений параметров решетки определены относительные продольные деформации кристаллитов (см. таблицу): $a$-ориентированные кристаллиты испытывают де-

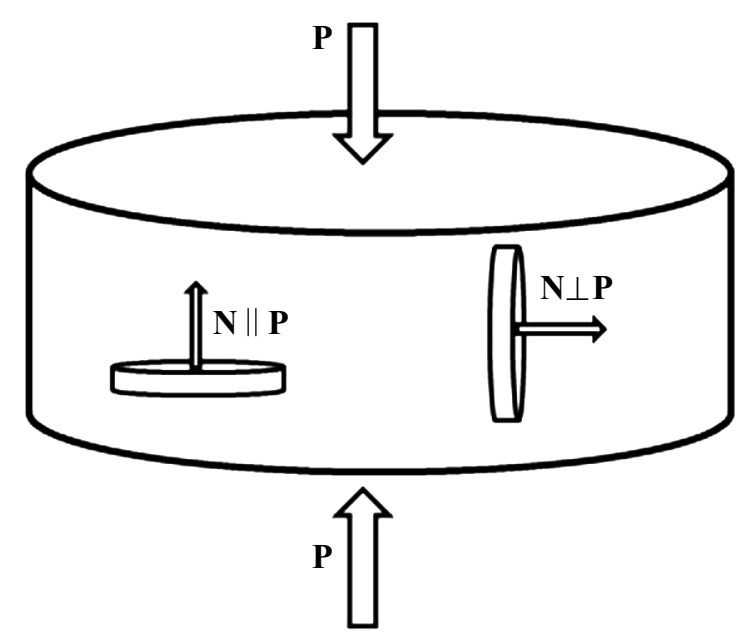

Pис. 1. Схема разрезки блока горячепрессованной керамики $\mathrm{KSN}$. $P$ - ось давления горячего прессования (ось текстуры), $N$ - направление нормали к рабочей плоскости измерительного образца. 


$$
\begin{aligned}
& \mathbf{P} \\
& a \text {-oriented } h k 0 \square a=12.433 \AA \quad 00 l] c=3.940 \AA
\end{aligned}
$$

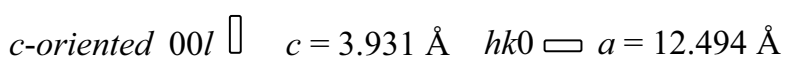

$$
\begin{aligned}
& \text { Powder: } \quad a=12.448 \AA \quad c=3.931 \AA \quad V=609.11 \AA^{3} \\
& \begin{array}{c}
\text { a-oriented } \\
V=609.04 \AA^{3}
\end{array} \varepsilon_{33} \leftarrow{\underset{\uparrow}{\downarrow}}_{\varepsilon_{11}}^{\varepsilon_{11}} \rightarrow \varepsilon_{33} \begin{array}{c}
c \text {-oriented } \\
V=613.58 \AA^{3}
\end{array} \varepsilon_{11} \leftarrow \square \rightarrow \varepsilon_{11}
\end{aligned}
$$

Рис. 2. Параметры решетки и деформации $a$ - и $c$-ориентированных кристаллитов. $P$ - ось давления.

Параметры решетки и относительные продольные деформации кристаллитов в керамических измерительных образцах KSN (относительные продольные деформации рассчитаны как $\varepsilon_{11}=\left(a_{t}-a_{i s o}\right) / a_{i s o}, \varepsilon_{33}=\left(c_{t}-c_{i s o}\right) / c_{\text {iso }}$; индексами iso и $t$ обозначены параметры изотропного образца и текстурированной керамики)

\begin{tabular}{c|c|c|c|c}
\hline Тип кристаллитов & $a, \AA$ & $c, \AA$ & $\varepsilon_{11}$ & $\varepsilon_{33}$ \\
\hline $\begin{array}{c}\text { KSN_iso } \\
c \text {-ориентированные } \\
\text { кристаллиты }\end{array}$ & 12.448 & 3.931 & & \\
$\begin{array}{c}a \text {-ориентированные } \\
\text { кристаллиты }\end{array}$ & 12.494 & 3.931 & 0.0037 & 0 \\
& 12.433 & 3.940 & -0.0012 & 0.0023
\end{tabular}

формации растяжения вдоль оси $c$ и сжатия вдоль оси $a$ без изменения объема, а $c$-ориентированные растянуты вдоль оси $a$ с увеличением объема ячейки; их относительные продольные деформации $\varepsilon_{33}$ увеличиваются с температурой и в области фазового перехода достигают максимальных значений.

Наличие текстуры, анизотропных деформаций кристаллитов и выявленные в [10] особенности структуры KSN должны сказаться на его объемных и поверхностных характеристиках, на пьезоотклике.

Значения объемного пьезомодуля $d_{33}$ текстурированных образцов измерены на установке d33-METER YE2730A (APC Instruments) и составляют $59 \mathrm{pC} / \mathrm{N}$ для $\mathrm{KSN} \_\mathrm{N} \| \mathrm{P}$ и $56 \mathrm{pC} / \mathrm{N}$ для KSN_N $\perp$ P. Неодинаковость значений объясняется нарушением изотропного распределения кристаллитов по ориентациям в текстурированных образцах. Кроме того, значительные величины деформаций $\varepsilon_{11}$ для $c$-ориентированных кристаллитов (рис. 2) могут обусловливать относительно высокий вклад компоненты $d_{31}$ в измеренное значение пьезомодуля.

Измерения пьезоотклика (PR) поверхности выполнены на сканирующем зондовом микроскопе (С3M) Veeco
Multimode VS в контактном режиме по стандартной методике. Верхняя поверхность образца полировалась до зеркального блеска, в нижнюю вжигался серебряный электрод. Для повышения чувствительности применен мягкий зонд (SCM-PIC, $k \approx 0.2 \mathrm{~N} / \mathrm{m}$, собственная частота $61.2 \mathrm{kHz}$ ). Из сравнения гистограмм рельефа на рис. 3, $a$ видно, что характерные размеры деталей поверхности трех образцов различаются незначительно: $7-18 \mathrm{~nm}$. При этом гистограммы PR (рис. $3, b$ и $c$ ) (так же как и соответствующие им изображения, не приведенные здесь ввиду краткого формата сообщения) отличаются от таковых для рельефа (рис. 3, $a$ ), зависимость амплитуды PR от амплитуды переменного напряжения $V_{a c}$ линейная, поэтому можно полагать, что изображения сформированы особенностями электромеханического отклика. Анализ частотной зависимости PR (аналогично [11]) показал, что при $50 \mathrm{kHz}$ амплитуда пьезоотклика текстурированных образцов становится гигантской, для изотропного образца этого не наблюдается. Это хорошо видно из сравнения масштабов на гистограммах (рис. $3, b, c$ ): отклик текстурированных образцов в 80-100 раз больше. Гистограммы PR (как и соответствующие им изображения) для $\mathrm{KSN} \_\mathrm{N} \| \mathrm{P}$ и $\mathrm{KSN} \_\mathrm{N} \perp \mathrm{P}$ (рис. $3, b$ и $c$ ) различаются из-за того, что их поверхности образуют по-разному ориентированные кристаллиты (рис. 2).

Поскольку амплитуда пьезоотклика прямо пропорциональна величине $d_{33}$ (вообще говоря, ее эффективному значению, но для оценки этого достаточно) [12], оценить изменения пьезоотклика удобно по отношениям величин пьезомодулей $d_{33}$, рассчитанных из амплитуд $D$ смещений поверхности и напряжений $V_{a c}$ для текстурированных и изотропного образцов $\left(d_{33}=D / V_{a c}[13]\right)$. Измерения выполнены с помощью одного и того же зонда, поэтому его чувствительность не влияет на результат. Для оценки $D$ достаточно средних значений, взятых по верхнему максимуму гистограмм (рис. $3, b$ и $c$ ), а поскольку $V_{a c}$ одинаковы для всех образцов $(2400 \mathrm{mV})$, относительные пьезомодули $d_{33}$ поверхности получаются как отношение значений $D: 62$ для $\mathrm{KSN} \_\mathrm{N} \| \mathrm{P}$ и 58 для $\mathrm{KSN} \_\mathrm{N} \perp \mathrm{P}$. Если полагать, что соотношение поверхностных пьезомодулей $d_{33}$ такое же, как объемных, то на поверхности его значение $\sim 3 \cdot 10^{3} \mathrm{pC} / \mathrm{N}$.

Различия величин относительных пьезомодулей обусловлены характером текстурирования керамики и распределением электрического поля зонда в поверхностном слое. Кроме того, обнаруженные нами специфические анизотропные деформации кристаллитов KSN (см. таблицу) должны существенно влиять на межзеренный интерфейс. На нем $[14,15]$ направление вектора спонтанной поляризации становится менее стабильным, а его чувствительность к внешнему полю повышается. В результате пьезоэлектрический отклик сильно увеличивается, причем его величина зависит от ориентации измерительного образца относительно оси текстуры, что соответствует различию относительных значений $d_{33}$ 

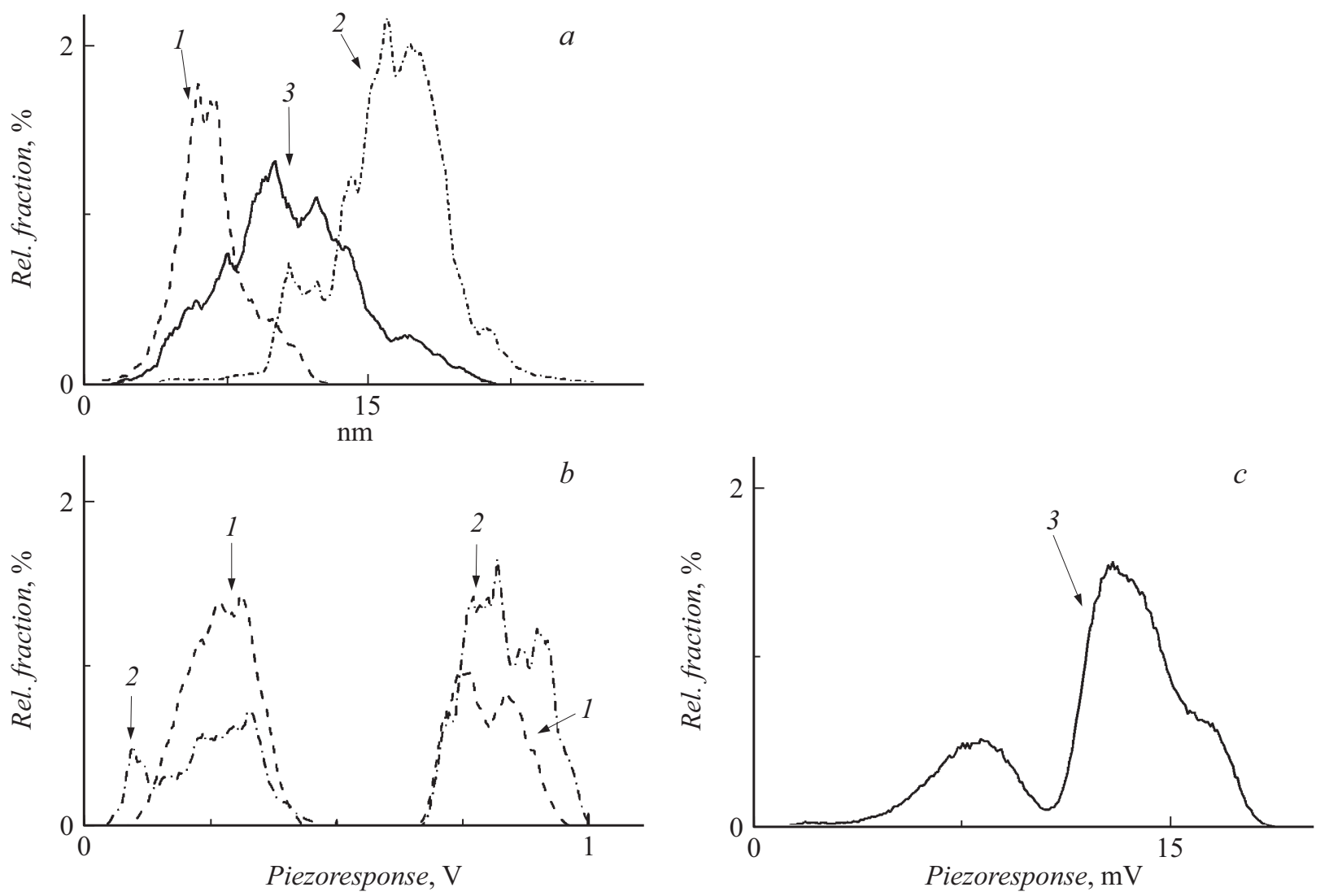

Рис. 3. Гистограммы распределения высот на СЗМ-сканах поверхности керамики $\mathrm{K}_{2} \mathrm{Sr}_{4} \mathrm{Nb}_{10} \mathrm{O}_{30}$. $a$ - топография, $b-$ пьезоотклик текстурированных образцов, $c-$ пьезоотклик нетекстурированного образца. 1 - KSN_N $\| \mathrm{P}, 2-\mathrm{KSN}$ _N $\perp$ P, $3-\mathrm{KSN} \_$iso. По оси ординат указана относительная доля участков поверхности, имеющих соответствующую амплитуду пьезоотклика.

для KSN_N $\|$ P и $\mathrm{KSN} \_\mathrm{N} \perp \mathrm{P}$. В [16] описано похожее увеличение пьезомодуля (более чем в 10 раз) для нанокомпозитных мезокристаллитов $\mathrm{BaTiO}_{3} / \mathrm{Bi}_{0.5} \mathrm{Na}_{0.5} \mathrm{TiO}_{3}$ из-за разницы $(2.6 \%)$ значений их постоянных решетки. В результате на гетероэпитаксиальной границе их раздела возникали деформации несогласования: решетка $\mathrm{BaTiO}_{3}$ сжата, а $\mathrm{Bi}_{0.5} \mathrm{Na}_{0.5} \mathrm{TiO}_{3}$ растянута. По-видимому, аналогичное влияние оказывают обнаруженные нами деформации $a$ - и $c$-ориентированных кристаллитов в KSN.

Еще одной причиной сильного увеличения электромеханического отклика поверхности может быть высокая плотность доменных стенок [17]. Кристаллам KSN свойственна высокая плотность ненасыщенных доменных стенок [8]. Чтобы учесть влияние всех названных факторов, в том числе анизотропных механических напряжений и полярных нанообластей [10], на гигантский пьезоотклик в KSN, необходимо отдельное рассмотрение, возможно, в рамках уточненной модели [17].

Таким образом, впервые для сегнетоэлектрикарелаксора со структурой ТВБ обнаружен гигантский пьезоотклик поверхности, сходный по величине с наблюдавшимся ранее в перовскитах. Возможной причиной этого могут быть специфические для текстурированной керамики $\mathrm{K}_{2} \mathrm{Sr}_{4} \mathrm{Nb}_{10} \mathrm{O}_{30}$ анизотропные механические напряжения.

\section{Финансирование работы}

Работа выполнена при поддержке проектной части государственного задания Минобрнауки РФ (тема № 3.1649.2017/4.6/ПЧ) и базовой части государственного задания Минобрнауки РФ (№ 3.6439.2017/БЧ).

\section{Конфликт интересов}

Авторы заявляют, что у них нет конфликта интересов.

\section{Список литературы}

[1] Tanaka S., Takahashi T., Furshima R., Makiya A., Uematsu K. // J. Ceram. Soc. Jpn. 2010. V. 118. P. 722-725.

[2] Zhu X., Fu M., Stennett M.C., Vilarinho P.M., Levin I., Randall C.A., Gardner J., Morrison F.D., Reaney I.M. // Chem. Mater. 2015. V. 27. P. 3250-3261.

[3] Ikeuchi Y., Takatsu H., Tassel C., Goto Y., Murakami T., Kageyama H. // Angew. Chem. Int. Ed. 2017. V. 56. P. 5770 5773.

[4] Филипьев В.С., Завьялов В.П., Бунина О.А., Гавриляченко С.В., Фесенко Е.Г. // ЖТФ. 1984. Т. 54. В. 3. С. 633-638.

[5] Liu L., Gao F., Zhang Y., Sun H. // J. Alloys Compd. 2014. V. 616. P. 293-299. 
[6] Duran C., Yildiz A., Dursun S., Mackey J., Sehirlioglu A. // Scripta Mater. 2016. V. 112. P. 114-117.

[7] Neurgaonkar R.R., Ho W.W., Cory W.K., Hall W.F., Cross L.E. // Ferroelectrics. 1983. V. 51. P. 185-191.

[8] Clarke R., Burfoot J.C. // J. Phys. D: Appl. Phys. 1975. V. 8. P. 1115-1120.

[9] Bunina O.A., Kuprina Yu.A., Raevski I.P., Knyazeva Ya.S., Raevskaya S.I., Chen H., Chou C.C., Titov V.V., Mezzane D., Sitalo E.I. // Ferroelectrics. 2016. V. 501. P. 145-153.

[10] Bunin M.A., Bunina O.A., Kuprina Yu.A., Raevski I.P., Inozemtsev S.V., Chen H., Raevskaya S.I., Sitalo E.I. // Ferroelectrics. 2018. V. 525. P. 18-27.

[11] Бунин М.А., Рыбянеи А.Н., Федоровский А.Е., Сухомлинов Д.И., Бунина О.А. // Изв. РАН. Сер. физ. 2016. Т. 80. № 11. C. $1580-1582$.

[12] Proksch R., Kalinin S. Piezoresponse force microscopy with Asylum Research AFM's. Oxford Instruments Asylum Research, Inc., 2015. P. 1-21.

[13] Hu D., Ma H., Tanaka Y., Zhao L., Feng Q. // Chem. Mater. 2015. V. 27. P. 4983-4994.

[14] Jiang A., Scott J., Lu H., Chen Z. // J. Appl. Phys. 2003. V. 93. P. $1180-1185$.

[15] Johnston K., Huang X., Neaton J.B., Rabe K.M. // Phys. Rev. B. 2005. V. 71. P. 100103.

[16] Zhang W., Ma H., Li S., Hu D., Kong X., Uemura S., Kusunose T., Feng Q. // Nanoscale. 2018. V. 10. P. 81968206.

[17] Sluka T., Tagantsev A., Damjanovic D., Gureev M., Setter N. // Nature Commun. 2012. V. 3. P. 748 (1-7). 